\section{Somatizar el castigo: un análisis de las experiencias de enfermedad y encierro penal de una mujer afroecuatoriana}

Somatizing punishment: An analysis of an Afro-Ecuadorian woman's experiences of illness and imprisonment

Vanessa Beltrán

\section{RESUMEN}

Este artículo indaga en las experiencias de enfermedad e internamiento penal de Analía Silva, una mujer afroecuatoriana y cofundadora de la organización Mujeres de Frente (MDF), quien fue condenada en múltiples ocasiones por el microtráfico de drogas y diagnosticada en el 2017, con una insuficiencia renal crónica. A través de la reconstrucción etnográfica del proceso de deterioro de su salud (2017-2020), se propone un análisis en torno a la pregunta; ¿Cómo se encarnan los procesos de desposesión y encierro penitenciario en la experiencia de enfermedad de una mujer empobrecida y racializada por el estado penal ecuatoriano? Se propone el argumento de que, en la intersección de sus experiencias de enfermedad y castigo penal, se encarnan los vínculos que unen la cárcel, la clínica y la calle como contextos expresivos del régimen securitario que ha guiado la gestión pública de la salud y el castigo penal en Ecuador, en las últimas décadas. El ingreso metodológico por la noción de cuerpo-territorio permitió develar la experiencia de padecimiento y encierro como síntomas de un sistema de organización social regido por la exclusión y el abandono selectivo del estado, que en los procesos de tratamiento de enfermedades como la insuficiencia renal crónica, se reproducen violencias normalizadas bajo la jerarquización del conocimiento y la instauración de ordenamiento moral castiga como indisciplina, la denuncia y la contestación y que en medio de contextos de sujeción, también es posible encontrar tejidos de politización con potencial transformador.

Palabras clave: Estado penal; género; salud; politización; violencia médica

\section{ABSTRACT}

This article inquiries into the experiences of illness and penal internment of Analía Silva, an Afro-Ecuadorian woman, and co-founder of the organization Mujeres de Frente (MDF), who was convicted multiple times for drug micro-trafficking and diagnosed in 2017 with chronic renal failure. Through the ethnographic reconstruction of her illness (20172020), the text discusses how the processes of dispossession and prison confinement are embodied in the experience of illness of an impoverished and racialized woman by the Ecuadorian penal state. The argument proposed is that, at the intersection of her experiences of illness and criminal punishment, the links that unite prison, the clinic, and the street as expressions of the government of the poor are expressed. The analysis based on the notion of body-territory, reveals the experience of suffering and confinement as symptoms of a social system that is organized by exclusion and selective abandonment of the state, that in the treatment of diseases such as renal failure, normalized violence are reproduced under the hierarchy of knowledge and the establishment of moral order that punishes as indiscipline, the complaint, and the contest and that in the midst of contexts of subjection, it is also possible to find politicizing networks with transformative potential.

Keywords: Penal state; gender; politicization; health; medical violence
RELIGACIÓN

REVISTA DE CIENCIAS SOCIALES Y HUMANIDADES JOURNAL OF SOCIAL SCIENCES AND HUMANITIES REVISTA DE CENCIAS SOCIAIS E HUMANAS

INFORMACIÓN:

https://doi.org/10.46652/rgn.v6i30.787 ISSN $2477-9083$

Vol. 6 No. 30, 2021. e210787

Quito, Ecuador

Enviado: 06 abril 2021

Aceptado: 09 septiembre 2021

Publicado: 29 octubre 2021

Publicación Continua

Sección Dossier | Peer Reviewed

\section{AUTORA:}

(D) Vanessa Beltrán

Universidad de Costa Rica - Costa Rica vanesa.beltran@ucr.ac.cr

\section{Conflicto de intereses}

La autora declara que no existe conflicto de interés posible.

Financiamiento

No existió asistencia financiera de partes externas al presente artículo.

Agradecimiento

Agradezco a Analía Silva y a la colectiva Mujeres de Frente por ser parte de este proceso de investigación acción feminista

Nota

Este artículo forma parte de la investigación de tesis para optar por la maestría en Género y Desarrollo de FLACSO-Ecuador.

ENTIDAD EDITORA 


\section{INTRODUCCIÓN}

Este artículo indaga en las experiencias de enfermedad e internamiento penal de Analía Silva, una mujer afroecuatoriana, cofundadora de la organización feminista y antipenitenciaria Mujeres de Frente (MDF), condenada en múltiples ocasiones por el microtráfico de drogas y diagnosticada en el 2017, con una insuficiencia renal crónica. A través de la reconstrucción del proceso de deterioro de su salud en los últimos tres años (2017-2020), se propone un análisis de las prácticas de violencia estatal que se ejercen sobre su cuerpo dentro de la institucionalidad penitenciaria y fuera de ella, en las dos clínicas público-privadas de atención renal a las que asiste en la ciudad de Quito, Ecuador.

A lo largo del texto se argumenta que, en la intersección de sus experiencias de enfermedad y castigo penal, se encarnan los vínculos que unen la cárcel, la clínica y la calle como contextos expresivos del régimen securitario que ha guiado la gestión pública de la salud y el castigo penal en Ecuador, en las últimas décadas. El cuerpo-enfermo adquiere un sentido analítico fundamental, para dar cuenta de los efectos encarnados de este régimen (Gago, 2019), caracterizado por el desfinanciamiento de los servicios sociales, el ataque permanente contra las economías informales, el racismo institucional y la reproducción de prácticas de exclusión contra los sectores urbanopopulares (Aguirre 2010, 2016; Coba 2010).

En la especificidad del territorio corporal de Analía, se configura un mapa de violencias estructurales y prácticas de resistencia política. Sus primeros quince años de vida, estuvieron atravesados por condiciones de empobrecimiento extremo, callejización temprana y sobre explotación laboral (Diario de campo, octubre 2018). Reaccionó a este entorno huyendo de su casa, buscando, en el comercio autónomo de drogas, una alternativa de sobrevivencia frente a la escasez de recursos y la desprotección de los programas sociales del estado.

A los diecisiete años, ingresó a prisión por primera vez, condenada por este delito. Recién se instauraba en el país el discurso norteamericano de la "guerra contra el narcotráfico", traducido en un endurecimiento de las leyes contra el tráfico de pequeñas cantidades y el fortalecimiento de la presencia policial en las calles del centro y sur de la capital. A partir de ese momento y por cuarenta años más, entró y salió de prisión, acumulando las sensaciones de desgaste, temor e incertidumbre que se producen como efecto de la sujeción al control penal intermitente (Silva en diario de campo, mayo 2018).

Vivió las transformaciones del modelo de gestión penitenciaria entre el periodo del neoliberalismo (1980-2007) y la Revolución Ciudadana (2007-2017) (Paladines, 2016; Aguirre et.al., 2020). Con su ingreso a uno de los megacomplejos penales inaugurados en la administración Correa Delgado (2013-2014), su condición de salud aceleró su deterioro, producto del aislamiento y la falta de acceso a recursos de limpieza, alimentación adecuada y agua potable (Plan V, 2018). En medio de la circulación por distintos centros penales, desde su juventud y hasta sus cincuenta y seis años, su riñón se fue debilitando hasta colapsar totalmente en el año 2017.

En este trayecto, fue testigo de lo que Wacquant ha elaborado como el "gobierno neoliberal de la pobreza" (Wacquant, 2010), que pasa, no solo por el fortalecimiento del brazo represor del estado, sino también, por el debilitamiento selectivo de su función social (Hernández, 2013; Segato, 2007; Wacquant, 2010). Entre el adentro y el afuera de la cárcel, vivió el estigma social del racismo y la exclusión sistemática de las esferas legales del trabajo. Siguiendo a Taussig (1980), el texto propone un abordaje de las experiencias de padecimiento de Analía como síntomas de todas estas condiciones, que, al reconstruir su historia de vida, se plasman como parte de la 
producción social de su enfermedad. En su caso, el cuerpo se presenta como un territorio de disputa política donde se pone en juego la lucha por la salud y la autonomía; una lucha que ha sido colectiva desde el momento en que se conformó la organización Mujeres de Frente.

A lo largo de este artículo, se presentan los principales hallazgos que emergen del proceso de acompañamiento a Analía y el análisis de sus experiencias de enfermedad de internamiento penal entre el 2017 y el 2020. Se inicia con un apartado metodológico que detalla las técnicas, categorías de investigación y fuentes de consulta que fueron abordadas a lo largo del proceso. Se sigue con la sección de resultados, expuestos en función de (1) el análisis de la enfermedad como síntoma de la desposesión y (2) la institucionalización del sufrimiento y la lucha colectiva por la vida. Se culmina con una reflexión final en torno a las preguntas que quedan por abordarse para futuros estudios en esta línea.

\section{METODOLOGÍA}

El proceso de investigación que dio vida a este texto expuso la complejidad ética y metodológica que supone aproximarse a los objetos de estudio desde el acompañamiento afectivo, el trabajo político militante y la propia subjetividad de la investigadora. Así, la reflexión colectiva y el diálogo en la diversidad y la desigualdad, fueron las premisas políticas y epistemológicas con las que se realizó la recopilación y análisis de datos (Salazar en Castro-Neira, 2019). Desde los principios de la investigación- acción colaborativa (Rappaport, 2007), se adquirió el compromiso de traducir los hallazgos de la investigación en insumos que aportaran a los procesos de denuncia pública de las múltiples violencias exacerbadas contra los sectores populares de la ciudad de Quitoespecíficamente aquellas vinculadas con el acceso a la salud.

La estrategia de recopilación de información fue trazada por el seguimiento etnográfico de las trayectorias de Analía entre el 2017-2020, con un énfasis en este último año, en que su salud empeoró en contexto de la pandemia por COVID-19. Esta aproximación participante se complementó con la vinculación de la investigadora en la asamblea de gestión de Mujeres de Frente, de ahí que las reflexiones propuestas sean resultado, no solo de la relación establecida con Analía, sino también de la experiencia de trabajo que supuso la militancia feminista entre mujeres distintas y desiguales. Involucrarse de lleno en las actividades cotidianas de la organización, potenció la comprensión de los contextos que se analizaban en el trabajo de campo, así como también, la necesidad de situar políticamente estos hallazgos en una perspectiva crítica, feminista y antipunitivista.

La recopilación de información se inscribe en el método etnográfico multilocal, propuesto por Marcus (2001), a través del seguimiento de trayectorias que en este caso, trascurrían entre la clínica, el encierro en casa y los hospitales. Se inscribe también en la línea de la autoetnografía analítica propuesta por Anderson (2006) por cuanto la investigadora se involucra personalmente en el grupo que participa del proceso de análisis, y se construyen insumos analíticos que informan a procesos políticos-históricos de mayor complejidad.

Por dos años (2018-2020), el trabajo organizativo estuvo distribuido entre el acompañamiento de Analía, la participación en sesiones colectivas de formación política, y, en contexto de pandemia, la organización y sostenimiento de la red de acompañamiento entre las mujeres de la organización. El diálogo en la desigualdad que emergía de estos espacios de encuentro, a partir del contacto con docentes, estudiantes, artistas, comerciantes autónomas, mujeres encarceladas y excarceladas, enriqueció la discusión de los hallazgos que se iban identificando en el proceso de investigación, poniendo en juego las experiencias de otras mujeres que también se reflejaban en la historia de Analía, y la amplia trayectoria de investigación- acción popular que colectivamente ha desarrollado la organización en los últimos quince años. 
En cuanto a las fuentes consultadas, se sistematizaron las publicaciones relacionadas con los centros de diálisis y su vínculo con el Ministerio de Salud Pública durante el periodo marzo-agosto 2020 publicadas por el diario ecuatoriano "El Comercio". Asimismo, se recurrió a las notas del diario de campo, en el que plasmaba los hallazgos de la aproximación etnográfica desarrollada, a través de la observación participante, que se desarrolló en la clínica de diálisis a la que asistía Analía tres veces por semana. De manera complementaria, se realizaron dos entrevistas abiertas a dos funcionarios de este centro de salud, y a una funcionaria del área de atención médica de la prisión en donde Analía descontó su última condena.

Esta información fue organizada bajo las categorías de violencia médica, enfermedad, y cuerpoterritorio (Gago, 2019). Se articuló el análisis de estas fuentes con el de los historiales clínicos y de internamiento penal de Analía y los resultados de un trabajo participativo en el que, a través del dibujo y palabras clave (registradas en el diario de campo), ella iba situando el marcaje de la enfermedad en su cuerpo. Estos datos fueron puestos en diálogo con los reportes del Observatorio Social del Ecuador (2020) y otras investigaciones que abordaban los procesos de desfinanciamiento del sistema público de salud, antes y durante el periodo de pandemia así como la instauración del neoliberalismo penal en Ecuador en medio de gobiernos progresistas.

En términos de las limitaciones de este abordaje metodológico, se reconoce la dificultad de acceder al detalle de los expedientes judiciales y de salud por tratarse de un grupo poblacional en condición de vulnerabilidad, la poca información estadística comparable en torno a las condiciones del sistema penitenciario ecuatoriano y particularmente en el tema de acceso a salud. Además, el contexto de confinamiento decretado por el gobierno ecuatoriano durante la pandemia dificultó el acercamiento regular al espacio de realización de la diálisis y, durante la última fase de investigación, exigió complementar el acompañamiento presencial que venía dándose con el seguimiento virtual, dado que, en medio de la emergencia, la investigadora salió repentinamente de Ecuador de regreso a Costa Rica, su país de origen. Estas limitaciones, así como las reflexiones éticas que atravesaron el ejercicio etnográfico son profundizadas en la sección de discusión.

\section{RESULTADOS}

El riñón comenzó a fallarle mucho antes de que llegara el diagnóstico oficial, en el año 2017. Inició como una molestia en su espalda que rápidamente se volvió crónica. Fue entonces cuando acudió al encargado del área médica de la cárcel de Cotopaxi y le insistió que necesitaba ser trasladada a un centro de atención médica. Llegó en ambulancia al Hospital General de Latacunga, donde permaneció internada cuatro días. Desde ese momento y hasta la actualidad, la vida de Analía depende de una máquina que limpia su sangre durante tres sesiones semanales de tres horas y media cada una. En su cuerpo- mapa han quedado marcadas las trayectorias que pasan, al menos, por tres centros penales y dos clínicas de diálisis, desde el momento en que se oficializó su diagnóstico y hasta el año 2020. Sin embargo, cuando se le consulta en qué momento fue que comenzó a sentirse enferma, Analía va mucho más atrás en el tiempo y afirma: “a mí la cárcel me terminó de enfermar. Y la clínica, también. Pero esta enfermedad que yo tengo empieza mucho antes, con la pobreza, la falta de agua y alimentos que impidiera que el riñón fallara (Silva, comunicación personal, 13 octubre 2018).

\subsection{La enfermedad como síntoma de la desposesión}

“Enfermedad renal crónica” (ERC)-como se le llama en el lenguaje técnico, es una patología clasificada como catastrófica por sus efectos bio-psicosociales, alta tasa de mortalidad, años de discapacidad y costos del tratamiento. Según el Ministerio de Salud Pública del Ecuador, la 
ERC es la cuarta causa de mortalidad ajustada por edad, por encima del cáncer y la diabetes. Es reconocida como una de las enfermedades más complejas de tratar por "la referencia tardía o en estadios avanzados, ausencia de programas de identificación precoz en personas con factores de riesgo para ERC, falta de médicos especialistas y limitación de opciones terapéuticas"(Ministerio de Salud Pública del Ecuador, 2018, p.18).

Según la normativa médica internacional, esta condición entra en el grupo de padecimientos catastróficos. La idea de catástrofe da cuenta de la complejidad de la enfermedad no solo en términos físicos e individuales, sino también en relación con los contextos socioeconómicos, familiares y psicoafectivos desde donde se produce (Tobar 2014). De acuerdo con estudios epidemiológicas, la ERC afecta sobre todo, a los sectores más desposeídos, con bajos ingresos y acceso a educación, habitualmente vinculados a dinámicas de trabajo irregular y sobreexplotación laboral (Díaz et al., 2018).

Analía se refleja en estos grupos, por la alta precarización que ha caracterizado su historia de vida, entre el empobrecimiento extremo y la intermitente callejización:

Yo antes de caer presa la primera vez nunca vi ayuda social, ni comida, ni vivienda, ni servicios de salud, inada! A nosotros en la calle lo único que nos llegaba eran las batidas policiales que detenían a cualquier indígena o a cualquier negro diciendo que éramos delincuentes. Cada quien tenía que ver cómo sobrevivía, de dónde agarraba comida, el agua, el abrigo para el frío... Porque del estado, solo el castigo (Silva, en diario de campo, octubre 2019).

En los primeros dos años de tratamiento, una vez oficializado su diagnóstico, circuló entre clínicas de atención médica, hospitales y centros de internamiento penal, como lo muestra la figura 1. Sus condenas variaban según la forma en que había ocurrido la detención y la cantidad de droga decomisada, pero por lo general, duraba entre uno y medio y dos años. Analía ha solicitado los beneficios de reducción de la pena por buen comportamiento y ejecución de más de la mitad de su condena varias veces. La última vez que salió libre, en el 2019, fue gracias a la política de unificación de penas que le benefició para salir antes de lo previsto.

Figura 1. Cronología de la enfermedad de Analía en relación con el contexto penitenciario y de atención médica

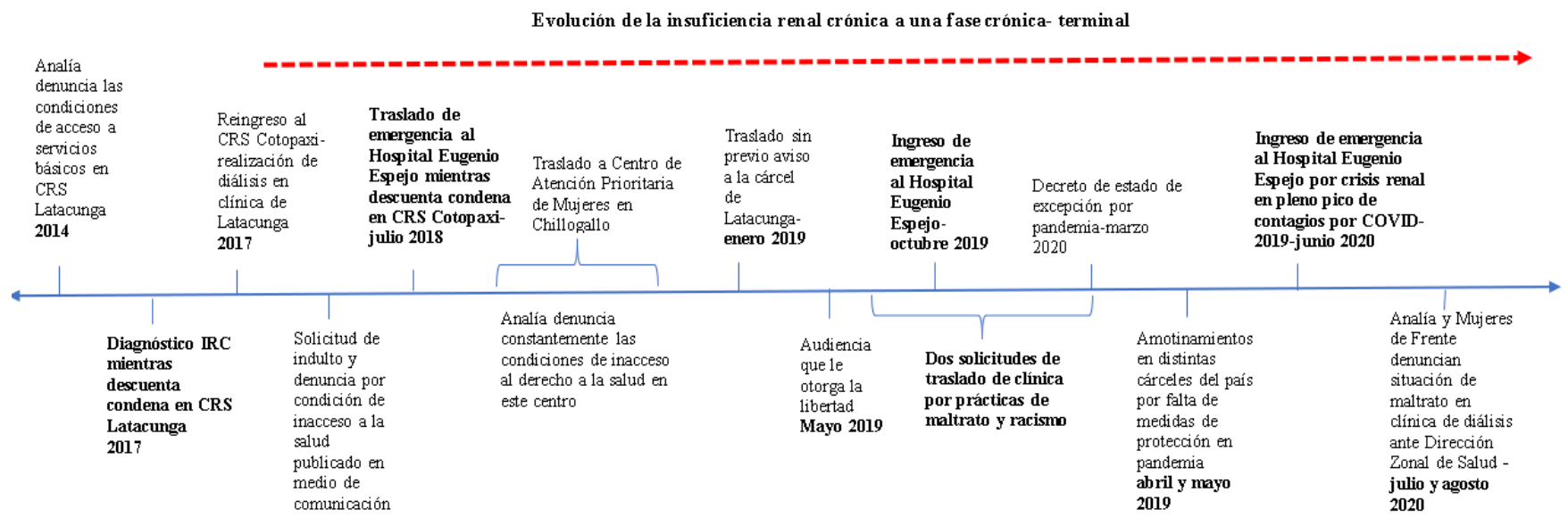

Fuente: datos de la investigación 
Los pinchazos, el catéter, los moretones acumulados alrededor de la clavícula y la sensación constante de fatiga muscular la han acompañado por más de tres años. En una primera etapa, vivió su tratamiento en la cárcel de Latacunga, lidiando con las condiciones generalizadas de inacceso a agua potable, alimentación adecuada y salud que ya han denunciado las personas privadas de libertad y organizaciones de derechos humanos (Comité Permanente por la defensa de los derechos humanos, 2019).

Entre el 2017 y el 2018 tuvo dos emergencias médicas que la llevaron a solicitar la imposición de medidas cautelares contra este centro penal y su inmediato traslado al Centro de Atención Prioritaria de Mujeres en Chillogallo- reclusorio que alberga mujeres con hijos e hijas menores de tres años, y población de mujeres con condición de vulnerabilidad. Era la época de transición entre las Administraciones de Correa Delgado (2007-2017) y Lenin Moreno (2017-2021). Para ese momento la administración de la justicia en Ecuador señalaba una disminución presupuestaria preocupante, agravada por el desfinanciamiento del sistema de salud público que se encargaba de gestionar la dimensión sanitaria de las prisiones (Báez, 2020; Comité Permanente por la defensa de los derechos humanos, 2019). Escasez de medicamentos, poco personal especializado para atender las demandas de salud en los centros, y la tercerización de los servicios de diálisis hacia el sector privado, eran las condiciones en las que Analía enfrentó los primeros años de su enfermedad, entre el adentro y el afuera de prisión.

Salió libre por última vez en el 2019. Pidió al Ministerio de Salud trasladarse de clínica en dos ocasiones por denuncias de maltrato y discriminación:

Me ponen el catéter mal dos o tres veces, y no te explican nada. A ellos no les importa el hueco que le dejan al cuerpo, dos o tres, no importa; a ellos solo les interesa cobrar el cheque a fin de mes. A mí no me preguntan ni cómo me siento. A los señores que son más blancos sí los tratan mejor. Les dan el refrigerio para que se lo coman ahí. Pero a mí no, me lo dan para que me lo lleve (Silva, comunicación personal, 14 de noviembre 2019).

Gracias a la red de Mujeres de Frente, se logró gestionar su ingreso a una nueva clínica de diálisis, en octubre 2019. Cinco meses después, en marzo del 2020, se decretó la emergencia sanitaria. El país había recibido la pandemia con un sistema hospitalario debilitado, una red de vigilancia epidemiológica recientemente desintegrada, y un nivel primario de atención médica en el que se había dejado de invertir durante la última década (Dávalos, 2016; Velasco et al., 2020). El gobierno optó por decretar el aislamiento social indefinido, haciendo eco del mensaje emitido por la Organización Mundial de la Salud (en adelante, OMS) en torno a la necesidad de controlar los focos de contagio.

Filas interminables en espera de una cama, hospitales saturados y falta de recursos para la compra de insumos de emergencia, fueron las condiciones en las que se desarrollaron los primeros meses de pandemia. La amplia presencia policial en espacios públicos resguardaba el cumplimiento de las medidas decretadas por el gobierno, como parte de un discurso oficial que homologaba el problema sanitario en clave de seguridad ciudadana. Quienes salían a la calle a vender su mercadería para alimentar a su familia eran abordados por la policía y los medios de comunicación como amenazas del orden público: “delincuentes”, “focos de contagios"- eran algunas de las expresiones reproducidas en los titulares de las noticias (Redacción El Universo, 2020). 
La inacción del estado frente al crecimiento de contagios en las prisiones y en los barrios populares de la ciudad, reflejaba la idea del abandono selectivo al que alude Wacquant cuando elabora su reflexión sobre el gobierno neoliberal de la pobreza (Wacquant, 2010). El discurso mediático sobre lo que acontecía en el sistema penitenciario y en las ciudadelas del sur de la capital, reproducía la narrativa del orden público y la seguridad nacional. Los amotinamientos en las cárceles por el derecho a la salud, las huelgas de hambre y el reclamo de las y los familiares de la población penal, se articulaba con el reclamo en las calles de grupos empobrecidos a los que la ayuda del estado nunca les llegaba y que, por el decreto de aislamiento, habían perdido la posibilidad de trabajar (Maisanche, 2020; Redacción El Universo, 2020).

Las personas con enfermedades crónicas como la insuficiencia renal (dentro y fuera de prisión), también se vieron significativamente afectadas. Años antes, el Ministerio de Salud había optado por consolidar un modelo de subcontratación del servicio de diálisis a clínicas privadas fiscalizadas por el estado (Ministerio de Salud Pública del Ecuador 2015, p.3), admitiendo su imposibilidad para atender la demanda creciente de la terapia de hemodiálisis. Conforme se acrecentó la crisis sanitaria, comenzaron a denunciarse la falta de recursos de las clínicas de diálisis, y las deudas impagas del estado a este sector (El Comercio, 2020). Se trataba de una problemática estructural que ponía a debatir el rumbo de la política social y sanitaria del país. La falta de recursos se había convertido en una excusa para librar de responsabilidad a los centros privados, fiscalizados por el estado; prueba de ello es que en medio de la emergencia el estado optó por destinar un amplio porcentaje de presupuesto al pago de su deuda externa, en lugar de destinarlo al abastecimiento de las instituciones de salud (Redacción Primicias, 2020).

Las venas de Analía se habían debilitado. Cada vez era más complicado asegurar la colocación de su catéter que comenzaba a dañarse de manera recurrente. Cada catéter era un pinchazo, un hueco más en su cuerpo; una cicatriz que quedaría por meses hasta que se fuera borrando entre la oscuridad de su piel. La posibilidad de cambiar a otra forma de diálisis parecía inviable, pues el tratamiento a nivel peritonial implicaba una inversión monetaria que la paciente debía asumir (Testimonio del médico tratante en diario de campo, agosto 2020). Mientras sus venas le permitieran seguirse dializando en el centro, Analía debía aguantar el dolor y continuar su terapia de esta forma.

Analía reconoce que en esta coyuntura su cuerpo experimento "un nuevo límite del dolor" que no puede ser explicado como un evento aislado, sino como producto de "una larga cadena de sufrimiento e incertidumbre" (Silva en diario de campo agosto 2019). Desde que se le diagnosticó su enfermedad, cada vez que ha sufrido un quebranto de salud, ha asistido a la clínica o al hospital para recibir atención. Salvo en dos ocasiones que fue internada de emergencia (2019 y 2020), el personal de la clínica y el hospital le han reiterado que, a falta de presentar síntomas de gravedad, debe regresar a su casa.

En una de las visitas que se realizaron a la clínica de diálisis con motivo de una de sus más recientes crisis de salud, el equipo técnico señaló que las señales que presentaba el cuerpo de Analía eran catalogadas como "efectos psicológicos de la pandemia" que podían mitigarse si "ella se tranquilizaba" (funcionario de la clínica en diario de campo, mayo 2020). Tal y como expresaban los médicos de la clínica "hay demasiados pacientes experimentando estas señales y no podemos atenderlos como quisiéramos porque no tenemos los recursos suficientes y porque no están asociados a su insuficiencia renal" (médico tratante en diario de campo, mayo 2020). Cuando buscaba con quien quejarse de la falta de atención especializada en la clínica, Analía no encontraba respuesta: el vínculo público-privado bajo el cual se regía aquel centro de salud 
dejaba abierta una zona difusa para la exigencia de protección estatal y garantía de acceso a sus derechos básicos.

De acuerdo con Taussig (1980), se entiende por síntoma, la expresión de un problema latente de mayor complejidad. El síntoma era el deterioro físico de Analía, conforme transitaba por las distintas esferas de sujeción estatal ya mencionadas. Lo que para los médicos se reducía a un padecimiento explicado por factores genéticos, para Analía era el reflejo de una trayectoria de vida resistiendo la precariedad y el abandono selectivo del estado. En uno de los últimos encuentros para trabajar sobre la historia de las cicatrices en su cuerpo, mencionó:

\footnotetext{
Estas marcas que yo llevo en el pecho son de mi nacimiento. Nací entre piedras, cuando mi madre caminaba en la romería de la Virgen del Quinche. Desde ahí, he pasado moviéndome, huyendo, resistiendo. Eso va dejando marcas en tu cuerpo. Son las marcas de la indolencia (Silva en diario de campo, abril 2020).
}

\subsection{La institucionalización del sufrimiento y la reivindicación colectiva de la vida}

Si hay algo que evidencia la trayectoria de Analía, dentro y fuera de prisión, la condena, como una condición que culmina al salir de la cárcel, es relativa al contexto en el que se viva el egreso, pues en su caso, el castigo se reconfigura en escenarios diversos que no se circunscriben exclusivamente al espacio penitenciario. A raíz de esta reflexión se discutió con Analía sobre el miedo, la enfermedad y la muerte. Estas tres palabras habían aparecido de manera reiterada en sus relatos, mucho antes de que se instalara la amenaza por COVID-19. Sin embargo, su narración había cambiado: la memoria de vivir la cárcel enferma había perdido valor frente a los recuerdos más recientes en donde ella señalaba haber sentido un miedo inminente de morir.

Por más que insistía en colocar estas preocupaciones en los espacios de encuentro con médicos y enfermeros, no había forma de que sus argumentos fueran colocados en el mismo rango de legitimidad que aquellos que elaboraba el personal técnico con base en los exámenes físicos que le realizaban periódicamente (Taussig, 1980). Constantemente se le abordaba con un tono condescendiente que la colocaba en el lugar de la que no conoce, la mujer pobre, excarcelada y enferma, cuyo cuerpo sobreviviría solo si se sometía al régimen propuesto:

\footnotetext{
Yo necesito que usted me le ayude a la señora para que entienda que tiene que seguir el tratamiento. Ella, pobrecita, está muy sola, y se ve que ha tenido una vida muy dura. Yo sé que detrás de ese carácter es que ella es una mujer muy frágil. Ayúdela, explíquele bien para que entienda que lo que hacemos aquí es por su salud (Médico tratante en diario de campo, enero 2020).
}

La salud era comprendida por las autoridades de las clínicas de atención médica en función de criterios técnico-especializados que dejaban por fuera las sensaciones, que señalaban los pacientes. Esto aparecía en los documentos que conforman su historia clínica unificada, no solo durante su último periodo en libertad, sino también en los periodos en los que estuvo recluida en centros penales. El grueso de este documento es acaparado por los resultados de los exámenes físicos, y las discusiones técnicas entre los equipos médicos para decidir el rumbo de sus intervenciones. Al margen y de forma aislada, quedaban plasmadas las notas de enfermería que incorporan al diagnóstico las vivencias y testimonios que Analía compartía. Aquella dimensión 
de la salud- afectiva y emocional, quedaba totalmente relegada al criterio técnico para definir el nivel de prioridad en la atención.

La memoria se activa cada vez que toca las cicatrices del brazo y la base del cuello. Sus venas están cada vez más delgadas, eso explica por qué cada vez la incisión para el ingreso del catéter requiere de mayor nivel de especialización (Testimonio de un médico de la clínica, comunicación personal 17 de enero 2020). Cada vez es más difícil resistir el dolor porque han sido ya muchos años de padecerlo, pero cuando mira su cuerpo en el espejo, reafirma su intención de resistir una intervención médica más; su color de piel es oscura, "es negra y es sinónimo de fuerza” (Silva en diario de campo abril 2020).

En los textos de denuncia que ha escrito con ayuda de compañeras militantes, Analía reafirma su negritud como una condición que, al mismo tiempo que la avergüenza, le potencia la necesidad de resistir. La cárcel, la calle y la clínica son espacios que han moldeado su capacidad de reproducir un discurso en el que estratégicamente se coloca como víctima, al mismo tiempo que produce un relato beligerante, crítico, que lejos de ubicarla en una posición de sujeción pasiva, la coloca en un lugar de resistencia. Calcula sus posturas y prevé escenarios de conflicto con la precisión de quien lleva años de moverse en la esfera del debate público:

Yo no hablo de justicia, yo hablo de (in)justicia, porque todo lo que hace el estado en nombre de la justicia, a nosotros los pobres nos termina castigando. Es solo justicia cuando eres del norte de Quito, eres blanco o mestizo y tienes dinero para pagar un buen abogado o un buen hospital donde te aseguran atención buena. A los demás esa justicia solo nos deja más pobres (Testimonio de Analía, diario de campo, setiembre 2019).

Desde que se conformó Mujeres de Frente, Analía ha conducido acciones de protesta contra la injusticia, tanto dentro como fuera de prisión. Entre el 2004 y el 2006 participó en el proceso de co-escritura feminista liderado por la organización a lo interno de la cárcel del Inca, en donde relató las prácticas de violencia y la condición de inacceso a la salud que se vive estando dentro (Mujeres de Frente 2004, 2006). Participó de la Organización de Reincidentes (2007) y más adelante, de la Organización de Familiares y Amigos de la Gente en Prisión (2014). Conversaba con sus compañeras de celda sobre la posibilidad de redactar notas a mano para denunciar la falta de personal médico y luego, estando en libertad, organizó varias reuniones con el equipo técnico de las clínicas de diálisis a las que asistía para reclamar su acceso a un tratamiento de calidad y con calidez, como lo establece la Ley General de Salud del Ecuador (2015). Cada una de esas acciones era producida a la luz de algún evento previo que le había generado dolor: la mala colocación del catéter, la falta de personal para asegurar su tratamiento dentro de prisión, la falta de alimentación adecuada y el maltrato de los funcionarios de las clínicas que se negaban a darle información detallada de su estado de salud (Diario de campo, enero-julio 2020). De cada crisis, emergía una nueva propuesta de contestación al régimen médico.

El recorrido de militancia ha sido central para producir esta postura política. Por más de quince años se había vinculado a aquel colectivo que integraba docentes, estudiantes, artistas, trabajadoras sexuales, trabajadoras del hogar, mujeres encarceladas y excarceladas y comerciantes autónomas de la calle. Las reconocía como su familia, porque junto con ellas, había construido un lenguaje político propio desde donde podía contestar las violencias que atravesaban sus trayectorias de vida. En aquella red de mujeres diversas, había conocido a otras compañeras, como Gloria, como Marianita, Margarita y Yolanda, con quienes, en contexto de pandemia, sostuvo un proceso de 
formación en salud comunitaria desde y para las necesidades de las compañeras que conformaban la organización.

A partir de mayo del 2020, más de treinta mujeres se conectaban en las dos sesiones semanales virtuales en las que se discutía, a través de dibujos y actividades pensadas para la diversidad de participantes sobre qué era el coronavirus, qué peligros tenía para la salud individual, familiar y comunitaria, y qué se podía hacer para prevenirlo. Sobre esta base se construyó un currículo formativo de cinco semanas en las que los miércoles y viernes se discutían los temas desarrollados en la cartilla, complementados con un sistema de acompañamiento entre las mujeres en el que cada una estaba pendiente vía WhatsApp, de al menos, otra compañera. Los temas propuestos en el currículo del curso suponían una adaptación de las medidas de prevención y cuidado contra la enfermedad, a los contextos específicos de estas familias. Era, la respuesta organizativa frente a la política securitaria que desplegaba el estado y que no alcanzaba las complejas realidades de quienes sostienen las redes populares de comercio autónomo callejero, en la ciudad de Quito.

Para Analía, estos espacios de encuentro colectivo habían significado salud en medio de la enfermedad, posibilidades de gestionar la vida en pleno contexto de escasez y desposesión. Así lo señaló en un texto coescrito por ella para la tercera edición de la Revista Sitiadas, una publicación de Mujeres de Frente en momentos de pandemia:

Mi familia, son ustedes. Las amigas, con las que peleo para tener al menos un poco de esa dignidad de la que hablo y no conozco bien. Con las que sigo luchando. ¿Cómo voy a dejar de luchar si es mi cuerpo? A veces como que te rindes, como que le haces caso a lo que te dicen los doctores y dices: "Ya estoy cansada". Pero luego también ves a la gente que quieres, a mi nieto el Martín, a ustedes compañeras, y te levantas. No por la vida que tienes sino por la que quieres (Silva en Mujeres de Frente, 2020).

\section{DISCUSIÓN}

Al ahondar en la historia de la enfermedad de Analía, se pudo comprender (1) cómo en su experiencia de padecimiento y encierro se expresan los síntomas (Taussig, 1980) de sistema de organización social que se sostiene de la exclusión, y el abandono selectivo del estado en función de las condiciones de raza, género y clase, (2) que en los procesos de tratamiento de estas condiciones, -clasificadas por el lenguaje técnico como "catastróficas," se reproducen violencias normalizadas bajo la jerarquización del conocimiento y la instauración de ordenamiento moral castiga como indisciplina, la denuncia y la contestación y (3) que en medio de contextos de sujeción, también es posible encontrar tejidos de politización con potencial transformador.

En el cuerpo-territorio de Analía se imprimen estas desigualdades, como continuidad de la exclusión que ha padecido entre la cárcel, la clínica y la calle. La continuidad es histórica y se revela en términos de la construcción de subjetividades punibles, sobre las cuales el estado, desde la gestión de la salud y el castigo, ha legitimado su (des)responsabilización (Aguirre, 2016; Coba, 2010). En su trayectoria de encierro y padecimiento, se evidencia cómo el cuerpo es tomado como un territorio de conquista, espacio histórico abierto a los vínculos y a la memoria, que se encarna no solo espacio, sino la pérdida de autonomía sobre él (Gago, 2019; Cruz, 2018). Desde el cuerpo-territorio es posible comprender cómo se encarna la desposesión de los medios de vida, y de qué manera el padecimiento se presenta como síntoma de este proceso.

Siguiendo a Gago (2019), ahí donde el estado se muestra como una totalidad impenetrable, se producen tejidos colectivos porosos, efímeros, y heterogéneos que se han afianzado en las 
alianzas populares para problematizar los complejos vectores de las violencias estructurales y potenciar la construcción de otros vínculos sociales tendientes a reconstruir el ya lastimado, tejido social urbano. Como bien lo refleja el proceso de articulación de una red de formación en salud desde Mujeres de Frente, en la que activamente participa Analía, la politización del padecimiento se despliega ahí donde el poder del estado y el saber médico confluyen para intentar sostener el estatus de subordinación de aquellas poblaciones reducidas a una condición de marginalidad. La manera en cómo Analía construye una crítica de la justicia a partir de su propia experiencia, que resulta de un proceso colectivo que supera su individualidad y la pone en diálogo con otras personas con quienes discute, piensa e interpela a las instituciones con las que se relaciona.

En este conjunto de experiencias se producen las condiciones de posibilidad para transformar el dolor, en un saber (Ahmed, 2015; Aranguren-Romero, 2010). La suma de escenarios de abuso, violencia, maltrato y abandono institucional son reconfigurados como núcleos de un discurso político situado en las múltiples condiciones de desigualdad que atraviesan su vida y desde donde despliega la potencia por la autonomía de su cuerpo-territorio. En este encadenamiento de experiencias, Analía transita entre el control estatal de su cuerpo, y el abandono al que es dejado cuando requiere de los servicios sociales y de salud para mantenerse con vida.

Si el engranaje institucional y los efectos multidimensionales del estado penal ecuatoriano se han centrado en el estudio de la esfera penitenciaria (Coba, 2015; Pontón, 2007), es pertinente ampliar el lente analítico fuera de ella, para alcanzar a ver la manera en que "el gobierno neoliberal de la pobreza"- como lo expone Wacquant (2010) copta ahora instituciones que desbordan lo penal y se inscriben en los ámbitos de la salud pública y la seguridad social. La categoría cuerpoterritorio adquiere una utilidad significativa en este propósito, porque devela el vínculo indisoluble entre el cuerpo y el espacio, la producción de subjetividad y procesos sociales e históricos más amplios, desde donde es posible analizar los paralelismos, rupturas y continuidades del tránsito entre instituciones de intervención penal y de atención médica. En ese sentido, el artículo abona al debate planteando la necesidad académica y política de continuar profundizando en la complejidad de lo que se conoce como el estado penal y el gobierno de la desposesión.

En la investigación planteada desde el acompañamiento, la autorreflexión y la colectividad, se agrieta también el distanciamiento entre la persona que investiga y quien es sujeta de análisis (Salazar, 2019; Anderson, 2006). Emprender este proceso fue asumir el hecho de que la mirada de la investigadora iba a ser confrontada por otra(s) cuyos intereses debían ser parte del diálogo de saberes que se pretendía construir; con las mujeres de la organización, con Analía y también con los y las funcionarias públicas con las que se realizaron intercambios. Ciertamente, las tensiones que podían emerger de ese encuentro de miradas, no se disolvían en una promesa de acompañamiento comprometido, transparente y horizontal. No era posible, justamente porque eran expresión y efecto de aquellos procesos históricos que en el camino se discutieron con Analía , desde donde se ha legitimado la jerarquía de saberes y el imaginario colonizador de que, quien investiga, escucha, sigue y analiza a sujetos desposeídos de autonomía, recursos y-o conciencia de su lugar en el mundo (Salazar en Castro-Neira 2019).

Fue al poner las vulnerabilidades propias y compartidas en juego, que se construyó un vínculo de afecto que trascendió la etapa de trabajo de campo y se extendió como tejido de amistad y militancia feminista transnacional. En ese sentido, se agrietó también la noción de transformación social como un proceso homogéneo y lineal; desde los feminismos populares y los tejidos de sobrevivencia gestados en medio de la precariedad, se tranzan procesos que discuten, revierten y recrean condiciones de posibilidad para una vida que merezca ser vivida. 


\section{CONCLUSIONES}

No hay duda de que los efectos de la experiencia en prisión y de padecimiento, se cargan en el cuerpo. En el caso de Analía, este proceso de encarnación se traduce en la evolución de una insuficiencia renal crónica que avanza hacia una fase terminal. Los diagnósticos han apuntado siempre hacia un origen genético que poco se vincula con el tránsito de ella entre el afuera y el adentro de la cárcel. Sin embargo, al analizar su testimonio y vincularlo a los contextos institucionales, políticos y económicos en los que este relato se produce, parece importante interpelar el discurso médico y preguntarse ¿Cómo se somatiza la (in)justicia?

Desde el 2017 y hasta la actualidad, el vínculo de Analía con los sistemas de salud y de justicia del Ecuador han develado las prácticas de violencia y exclusión que se legitiman desde el accionar estatal. Su experiencia da cuenta de la producción pública de cuerpos que, estigmatizados por el empobrecimiento, la racialización y el contacto con la pena de cárcel, merecen ser abandonados por la institucionalidad pública. Las barreras que le impiden acceder a sus derechos surgen, no solo de su experiencia en prisión, sino también de la vida en la calle, donde paradójicamente la ley le reconoce el pleno ejercicio de sus derechos en su calidad de ciudadana. Circular entre estos dos espacios, le ha hecho cuestionar la frontera entre lo que la política penal y penitenciaria separa como el adentro y el afuera de la cárcel.

En contexto de pandemia, cuando estas condiciones se ven exacerbadas por las medidas sanitarias implementadas por el gobierno, fue a través del acompañamiento conjunto que fue posible captar estas experiencias, donde se materializan- al mismo que se agrietan, las violencias de estado. No fue sino a través del vínculo político, construido con Analía y Mujeres de Frentela organización de la cual es cofundadora, que emergió la idea de que, al preguntarse por las experiencias de padecimiento de una mujer afroecuatoriana en contextos de encierro, se planteaba, necesariamente, una pregunta por la manera en que el estado gestiona de manera diferenciada el control y abandono de la vida de las poblaciones. No fue sino a través de la reflexión constante y colectiva, que se evidencia que, en medio del despliegue exacerbado del control social- en la prisión, en las calles y en la clínica, se gestionaban territorios de politización.

El planteamiento de una metodología propuesta desde y para la acción, posibilitó llegar ahí donde los discursos estatales no han podido entrar; en el terreno que se gestiona en los márgenes de los informes institucionales y los estudios estadísticos. Sobre esa base, la investigación- acción sigue siendo una clave de ingreso vigente para el desarrollo de propuestas analíticas políticamente situadas y con potencial transformador. En ese sentido, quedan abiertos múltiples espacios para seguir sosteniendo procesos de investigación-acción, comprometidos con la recuperación de esos "otros" escenarios en donde la cotidianidad se vuelve espacio de lucha; desde donde se puede demostrar que el capitalismo, el patriarcado y el legado colonial que atraviesa nuestras sociedades no lo han roto todo. Como bien señala Analía: “¿Cómo voy a dejar de luchar si es mi cuerpo?" (Mujeres de Frente, 2020, p. 45).

\section{REFERENCIAS}

Aguirre, A. (2016). La delincuencia en Quito entre 1960 y 1980: discursos y prácticas de punición, transgresión y resistencia. [Tesis doctoral, Universidad Andina Simón Bolívar]. Biblios- e Archivo http://hdl.handle. net $/ 10644 / 5673$

Aguirre, A. (2019). Incivil y criminal: Quito como escenario de construcción estatal de la delincuencia entre los decenios 1960 y 1980. Universidad Andina Simón Bolívar. 
Aguirre, A., León, T., \& Ribadeneira, N. (2020). Sistema penitenciario y población penalizada durante la Revolución Ciudadana (2007-2017). Urvio, Revista Latinoamericana de Estudios de Seguridad, (27), 94110. https://doi.org/10.17141/urvio.27.2020.4303.

Ahmed, S. (2015). La política cultural de las emociones. Universidad Nacional Autónoma de México.

Anderson, L. (2006). Analytic Autoethnography. Journal of Contemporary Ethnography, 35(4), 373-395. https://doi.org/http://dx.doi.org/10.1177/0891241605280449.

Aranguren-Romero, J. P. (2010). De un dolor a un saber : cuerpo, sufrimiento y memoria en los límites de la escritura. Papeles del CEIC. International Journal on Collective Identity Research, 2(63), 1-27.

Arteaga, É., Cuvi, J., \& Maldonado, X. (2017, mayo 28). Políticas neoliberales de salud en el gobierno de Correa. Salud y Derechos. https://cutt.ly/kRO72Hr

Báez, J. (2020, marzo 28). Los recortes cobran factura al Ecuador: La inversión en salud se redujó un 36\% en 2019. Unidad De Análisis Y Estudios De Coyuntura https://cutt.ly/vRO77P3

Boutron, C., \& Constant, C. (2013). Gendering Transnational Criminality: The Case of Women's Imprisonment in Peru. Signs: Journal of Women in Culture and Society, 46(1), 177-195.https://doi.org/10.1086/670827.

Cruz, T. (2018). Una mirada muy otra a los territorios-cuerpos femeninos. Solar, 12(1), 35-46. https://doi. 10.20939/solar.2016.12.0103.

Chonata, J. F. (2012). Análisis de supervivencia de pacientes en hemodiálisis en el periodo 2000-2010 en el Centro de Diálisis Dialcentro. [ Tesis doctoral especialidad en nefrología Universidad San Francisco de Quito]. Biblios- e Archivo. http://repositorio.usfq.edu.ec/handle/2300o/4744.

Coba, L. (2010). Territorios del desarraigo: subjetividades penalizadas, entre la ley del padre y la ética materna. [Tesis doctoral, FLACSO-Ecuador]. Biblios- e Archivo http://hdl.handle.net/10469/3382.

Coba, L. (2015). Sitiadas: la criminalización de las pobres en Ecuador durante el neoliberalismo. FLACSO.

Comité Permanente por la Defensa de los Derechos Humanos. (2019, julio 31). Informe sobre crisis carcelaria en Ecuador, actualización. Comité Permanente por la Defensa de los Derechos Humanos. https://cutt. ly/eRO5jlg

Constant,C.(2016).Pensarlaviolencia delas mujeres.Laconstrucción de la figuradelincuente.Políticaycultura, 46, 145-162. http://www.scielo.org.mx/scielo.php?script=sci_arttext\&pid=S0188-77422016000200145

Darke, S., \& Karam, M. L. (2017). Las prisiones en América Latina. Revista Debate, 101, 53-71.

Dávalos, P. (2016). Salud Inc. Monopolio, ganancia y asimetrías de la información en el aseguramiento privado de la salud en el Ecuador. Pontificia Universidad Católica del Ecuador.

Díaz, A., Gómez, L. B., Robalino, V., \& Lucero P. (2018). Comportamiento epidemiológico de pacientes con insuficiencia renal crónica en Ecuador. Correo Científico Médico, 22(2), 312-324. http://scielo.sld.cu/ scielo.php?script=sci_arttext\&pid=S1560-43812018000200011

Fleetwood, J. (2016). Drug Mules . Prison Service Journal, 192(1), 3-8.

Gago, V. (2019). La potencia feminista o el deseo de cambiarlo todo.Tinta Limón.

Gago, V., \& Quiroga, N. (2014). Los comunes en femenino. Cuerpo y poder ante la expropiación de las economías para la vida. Revista Economía y Sociedad, 19(45), 1-18. https://doi.org/10.15359/eys.19-45.1

Guber, R. (2001). La etnografía: método, campo y reflexividad. Norma.

Hernández, A. (2013). ¿Del Estado multicultural al Estado penal? Mujeres indígenas presas y criminalización de la pobreza en México. En Sierra, M.T.(ed) Justicia, pueblos indígenas y violencia en México y Guatemala (299-338). CIESAS-FLACSO.

Ley General de Salud del Ecuador, 67/2015. Registro oficial suplemento 423, 22 de diciembre 2006. https:// cutt.ly/CRO5EIt

Maisanche, F. (2020, abril 21). Fiscalía investiga amotinamientos en las cárceles de Ambato y Puyo. El Comercio. https://cutt.ly/FRO5IMZ

Marcus, E. (2001). Etnografía en/del sistema mundo. El surgimiento de la etnografía multilocal. Revista Alteridades 11(22), 111-127. https://alteridades.izt.uam.mx/index.php/Alte/article/view/388 
Ministerio de Salud Pública del Ecuador. (2015 junio). Programa Nacional de Salud Renal. En Ministerio de Salud Púbica del Ecuador. https://cutt.ly/NRO5Sdm

Plan V. (2018, marzo 26). La cara cruel de la cárcel de Latacunga. Plan V. https://cutt.ly/pRO5FK8

Mujeres de Frente. (2004, septiembre) Sitiadas 1. Un trabajo de mujeres en situación. https://mujeresdefrente. org/wp-content/uploads/2018/10/Sitiadas-1.pdf.

Mujeres de Frente. (2006). Sitiadas 2. Un trabajo de mujeres en situación. https://mujeresdefrente.org/wpcontent/uploads/2020/11/Sitiadas_compressed-1-1.pdf.

Mujeres de Frente. (2020). Sitiadas 3: reflexiones sobre el estado punitivo y el sostenimiento de la vida sin estado. Fundación Rosa Luxemburgo.

Ponce, J. (2020, julio 30). Trabajadores de los centros de diálisis protestan por los meses impagos. Diario Expresso. https://cutt.ly/qRPqncL

Pontón, J. (2007). Cárceles del Ecuador: los efectos de la criminalización por drogas. URVIO Revista Latinoamericana de Seguridad Ciudadana, 1(1), 55-73. https://doi.org/10.17141/urvio.1.2007.1053

Rappaport, J. (2007). Más allá de la escritura: la epistemología de la etnografía en colaboración. Revista Colombiana de Antropología, 43, 197-229.

Redacción El Universo (2020, marzo 10). El presidente Lenín Moreno anunció recorte en gasto público. El Universo. https://cutt.ly/JRPqvwC

Redacción Primicias. (2020, julio 4). Así funciona la base de beneficiarios de los kits de alimentos. Primicias. https://www.primicias.ec/noticias/sociedad/kits-alimentos-registro-social-coronavirus/.

Salazar, S. (2019). Afectividad y violencia: reflexiones en torno a una experiencia de trabajo de campo en Honduras y el sur de México. En Y. Castro. (coord) Antropología de la violencia, (pp. 183-212). Benemérita Universidad Autónoma de Puebla.

Segato, L. (2007). El color de las cárceles de América Latina: Apunte sobre la colonialidad de la justicia en un continente en deconstrucción. Nueva sociedad, 208, 142-161.

Taussig, M. (1980). "Reification and the consciousness of the patient". Social Science and Medicine. Part B Medical Anthropology, 14(1), 3-13. https://doi.org/10.1016/0160-7987(80)90035-6.

Teleamazonas Ecuador. (2020, julio 20). Moradores de Colinas del Norte piden la realización de pruebas para Covid-19. Teleamazonas Ecuador. https://www.youtube.com/watch?v=tEujmZu3Xg8.

Tobar, F. (2014). Respuestas a las enfermedades catastróficas. CIPPEC.

Velasco, M., Hurtado, F., \& Tapia, J. (2020). ¿Estaba preparado el sistema de salud para enfrentar la pandemia? Observatorio Social del Ecuador. https://www.covid19ecuador.org/post/salud-publica-pandemia-1.

Wacquant, L. (2010). Crafting the Neoliberal State: Workfare, Prisonfare, and Social Insecurity. Sociological Forum, 25(2), 197-220. https://doi.org/10.1111/j.1573-7861.2010.01173.x.

Zaragocín, S. (2019). Gendered Geographies of Elimination: Decolonial Feminist Geographies in Latin American Settler Contexts. Antipode, 51(1), 373-392. https://doi.org/10.1111/anti.12454

\footnotetext{
AUTORA

Vanessa Beltrán. Egresada de la Maestría en Género y Desarrollo de FLACSO-Ecuador. Licenciada en Ciencias Políticas de la Universidad de Costa Rica y actualmente docente e investigadora de la Escuela de Ciencias Políticas y el Centro de Investigación y Estudios Políticos de la Universidad de Costa Rica. Líneas de investigación: feminismos, cuerpo y subjetividades, contextos penitenciarios.
} 